\title{
Improvements in LDL particle size and distribution by short-term alternate day modified fasting in obese adults
}

\author{
Krista A. Varady ${ }^{1 *}$, Surabhi Bhutani ${ }^{1}$, Monica C. Klempel ${ }^{1}$ and Benoît Lamarche ${ }^{2}$ \\ ${ }^{1}$ Department of Kinesiology and Nutrition, University of Illinois at Chicago, 1919 West Taylor Street, Room 506F, \\ Chicago, IL 60612, USA \\ ${ }^{2}$ Nutraceuticals and Functional Foods Institute, University of Laval, Sainte-Foy, QC, Canada \\ (Received 13 April 2010 - Revised 2 June 2010 - Accepted 25 August 2010 - First published online 30 September 2010)
}

\begin{abstract}
Alternate day modified fasting (ADMF) beneficially modulates several indicators of CHD risk, but its effects on LDL particle size have never been tested. Accordingly, we examined the effects of ADMF on LDL particle size and distribution in obese adults. Sixteen obese subjects participated in a 10-week trial with three consecutive phases: (1) 2-week control phase; (2) 4-week ADMF controlled feeding phase; (3) 4-week ADMF self-selected feeding phase. After 8 weeks of diet, body weight and waist circumference were reduced $(P<0 \cdot 05)$ by $5.6($ SEM 0.9$) \mathrm{kg}$ and $4.0($ SEM 0.9$) \mathrm{cm}$, respectively. LDL-cholesterol and TAG concentrations decreased $(P<0.05)$ by 25 (SEM 10$) \%$ and 32 (sem 6) \%, respectively. Peak LDL particle size increased $(P<0.05$ ) from 266 (sem 1) to 268 (sem 1) A. Additionally, the proportion of small LDL particles decreased $(P<0.05$ ) from 13 ( (АЕм 2 ) \% to 9 ( АЕм 3 ) \%, while the proportion of large LDL particles increased $(P<0 \cdot 05$ ) from 68 (SEM 4) \% to 76 (SEM 4) \% post-treatment. These findings suggest that ADMF is an effective diet strategy for increasing LDL particle size and decreasing the proportion of small, dense LDL particles in obese adults.
\end{abstract}

Key words: Alternate day fasting: LDL particle size: Cholesterol: CHD: Obese adults

A preponderance of small, dense LDL is associated with a 3- to 7-fold increase in CHD risk, independent of LDLcholesterol concentrations ${ }^{(1)}$. The mechanisms that link small LDL particles to atherogenesis are thought to include: long residence time in plasma, enhanced oxidisability and increased permeability through the endothelial barrier ${ }^{(2)}$. An increased proportion of small dense LDL is associated with moderate elevations in plasma TAG as well as low levels of HDL-cholesterol ${ }^{(2)}$. Carrying excess adipose tissue, particularly in the abdominal region, is also correlated to an increased proportion of small $\mathrm{LDL}^{(3)}$. Weight loss by means of energy restriction (ER) has been shown to increase LDL particle size, and thus reduce CHD risk in obese adults ${ }^{(4-6)}$.

Alternate day modified fasting (ADMF) is a form of dietary restriction that has been shown to help overweight and obese individuals lose weight and lower $\mathrm{CHD}$ risk $^{(7,8)}$. ADMF regimens consist of a 'feed day', where food intake ad libitum is permitted for $24 \mathrm{~h}$, alternated with a 'fast day', where energy intake is limited to $25 \%$ of needs for $24 \mathrm{~h}$. Although the data are limited, recent results indicate that ADMF for 8 weeks reduces body weight by
6-8\%, decreases waist circumference and lowers LDL-cholesterol and TAG concentrations by 10-25 and $30-40 \%$, respectively ${ }^{(7,8)}$. In view of these beneficial effects on plasma lipids, it is hypothesised that this diet therapy would also improve other key CHD risk factors, such as LDL particle size.

Accordingly, the objective of the present study was to examine the effects of ADMF on LDL particle size in obese subjects and to evaluate how these modulations of LDL diameter are related to changes in visceral fat mass and plasma lipid concentrations.

\section{Materials and methods}

\section{Subjects}

The present study was conducted according to the guidelines laid down in the Declaration of Helsinki, and all procedures involving human subjects were approved by the Office for the Protection of Research Subjects at the University of Illinois, Chicago. A written informed consent was obtained from all the subjects. The key inclusion criteria were as follows: age 35-65 years; BMI between

Abbreviations: ADM, alternate day modified fasting; ER, energy restriction. 
30 and $39.9 \mathrm{~kg} / \mathrm{m}^{2}$; weight stable for 3 months before the beginning of the study (i.e. $<5 \mathrm{~kg}$ weight loss or weight gain); non-diabetic; no history of CVD; lightly active (i.e. $<3 \mathrm{~h}$ /week of light-intensity exercise at 2.5-4.0 metabolic equivalents for 3 months before the study); non-smoker; not taking weight loss, lipid-lowering or glucose-lowering medications.

\section{Study design and alternate day modified fasting protocol}

Sixteen subjects participated in a 10 -week trial with three consecutive intervention phases: (1) 2-week pre-loss control phase; (2) 4-week weight loss/ADMF-controlled feeding phase; (3) 4-week weight loss/ADMF self-selected feeding phase. During phase 1 , all the participants maintained their usual eating habits in order to maintain body weight. During phase 2, subjects consumed $25 \%$ of their baseline energy needs on the fast day (between 12.00 and 2.00 hours) and then ate ad libitum on each alternate feed day. Baseline energy requirements were determined by the Mifflin equation ${ }^{(9)}$. During this controlled feeding phase, the subjects were provided with a energy-restricted meal on each fast day $(25 \% \mathrm{~kJ}$ as protein, $25 \% \mathrm{~kJ}$ as fat and $50 \% \mathrm{~kJ}$ as carbohydrate), while on each feed day, subjects ate ad libitum at home. During phase 3, the subjects continued the same ADMF regimen but were required to prepare all of their meals at home. During this self-selected feeding phase, subjects met weekly with the study dietitian to learn how to maintain the ADMF regimen on their own. Dietary adherence on the fast day was monitored using the 'Extra food log'. The subjects were asked to report any extra food item consumed on the fast day that did not comply with their prescribed plan using this log. If the log indicated that the subject ate an extra food item on a fast day, that day was labelled as 'not adherent'. If the log revealed that the subject did not eat any extra food item, that day was labelled as 'adherent'. Percentage adherence/week was calculated as follows:

$$
\begin{aligned}
\% \text { Adherence }= & \text { number of fast days adherent } / \\
& \text { number of fast days in the week } \times 100 .
\end{aligned}
$$

In addition, average energy intake was determined by collecting feed day food records ( 2 week days and 1 weekend day) during each phase. Fast day food records were collected during phase 3. Nutritionist Pro Software (version 4.3; Axxya Systems, Stafford, TX, USA) was used to calculate total daily intake of energy. Alcohol consumption was restricted to no more than one drink/d for women and no more than two drinks/d for men. Body weight was assessed at the research centre at the beginning of every week in light clothing without shoes. BMI was assessed as $\mathrm{kg} / \mathrm{m}^{2}$. Twelve hour fasting blood samples were collected between 07.00 and 09.00 hours at baseline (day 1), at the end of phase 1 (day 14), at the end of phase 2 (day 41: feed day and day 42: fast day) and at the end of phase 3 (day 69: feed day and day 70: fast day).

\section{Plasma lipids and LDL particle size}

Plasma total cholesterol, HDL-cholesterol and TAG concentrations were measured in duplicate using enzymatic kits (Biovision, Inc., Moutainview, CA, USA). The concentration of LDL-cholesterol was calculated using the Friedewald equation ${ }^{(10)}$. LDL particle size analysis was performed on whole plasma using non-denaturing 2-16\% polyacrylamide gradient gel electrophoresis, as described previously ${ }^{(11,12)}$. Peak and integrated LDL particle diameter was computed using the approach described by Tchernof et $a l{ }^{(12)}$. The relative proportion of small $\left(\mathrm{LDL}_{<255 \AA} \%\right)$, medium $\left(\mathrm{LDL}_{255-260 \AA} \%\right)$ and large $\left(\mathrm{LDL}_{>260} \AA\right.$ \%) particles was obtained by computing the relative area of the densiometric scan $(<255,255-260 \text { and }>260 \AA \text {, respectively })^{(12)}$. The absolute concentration of cholesterol among particles was estimated by multiplying the total plasma LDLcholesterol level by the relative proportion of small, medium and large particles.

\section{Statistics}

The results are presented as means with their standard errors. One-factor ANOVA was performed to determine an overall $P$ value for each variable, and the Bonferroni correction was used to assess the significance. Relationships between continuous variables were assessed by simple regression analyses as appropriate. Tests for normality were included in the model. Data were analysed by using SPSS software (version 17.0 for Mac OS X; SPSS, Inc., Chicago, IL, USA).

\section{Results}

\section{Baseline characteristics and weight loss}

Sixteen subjects (four men and twelve women) completed the entire 10-week trial. Four women were post-menopausal, while eight women were pre-menopausal. Six of the pre-menopausal women were taking oral contraceptives as a birth control method. The other two pre-menopausal women were using barrier methods as birth control. Completers were middle aged (46 (sEm 3) years), obese (BMI 34 (SEM 1) kg/m²), sedentary $(2 \cdot 4$ (sEM $0 \cdot 3) \mathrm{h} /$ week of physical activity) and borderline hypercholesterolaemic (LDL-cholesterol concentration 106 (sEM 100) mg/l). Dietary adherence on the fast day was high and stable over the course of the study (phase 2: 86 (SEM 4)\% adherence and phase 3: 89 (SEM 5)\% adherence). Average energy intake was as follows: phase 1, 8104 (SEm 748) kJ/d; phase 2 feed day, 7497 (SEM 953) kJ/d; phase 2 fast day, 1725 (sem 351) kJ/d; phase 3 feed day, 6882 (sem 782) kJ/d; phase 3 fast day, 2460 (sem 192) kJ/d. Body weight 


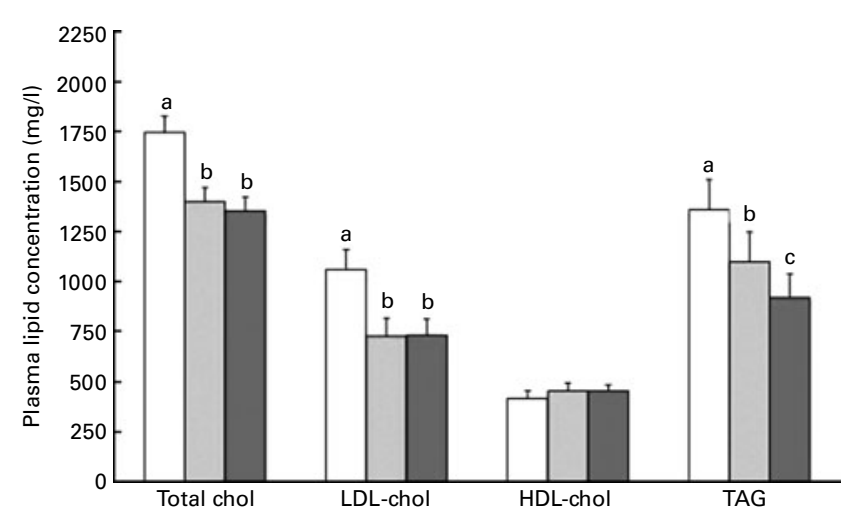

Fig. 1. Plasma lipid concentrations over the course of the trial. Values are reported as means with their standard errors, $n 16$ (days 41 and 42 values combined). ${ }^{a, b, c}$ Means with unlike superscript letters were significantly different between time points for separate lipid parameters, $P<0.05$ (one-factor ANOVA with Bonferroni analysis). ${ }^{a, b, c}$ Mean values with unlike superscript letters were not significantly different between time points for separate lipid parameters, $P<0.05$ (one-factor ANOVA with Bonferroni analysis). $\square$, Day 14; $\square$, days 41, 42; $\square$, days 69, 70. chol, Cholesterol.

decreased $(P<0.001)$ by $2 \cdot 8$ (SEM 0.5$) \mathrm{kg}$ after 4 weeks and by 5.6 (SEM 1.0) $\mathrm{kg}$ after 8 weeks of ADMF. BMI decreased from $33.7 \mathrm{~kg} / \mathrm{m}^{2}$ (baseline) to $31.4 \mathrm{~kg} / \mathrm{m}^{2}$ (post-treatment). Waist circumference did not change after 4 weeks of treatment, but decreased $(P<0.05)$ by $4.0($ SEM 0.9$) \mathrm{cm}$ after 8 weeks of ADMF.

\section{Plasma lipids and LDL particle size}

Total cholesterol, LDL-cholesterol and TAG concentrations decreased $(P<0.05)$ by 21 (SEM 4) \%, 25 (SEM 10) \% and 32 (sem 6) \%, respectively, from baseline to the end of the study. HDL-cholesterol concentrations were not affected by the intervention (Fig. 1). Peak and integrated LDL particle size increased $(P<0.05)$ after 8 weeks of ADMF (Table 1). The proportion of large LDL particles $(>260 \AA)$ increased $(P<0.05)$, while the proportion of small $(<255 \AA)$ and medium $(255-260 \AA)$ particles decreased $(P<0.05)$. The estimated concentration of cholesterol in large particles did not change, while the concentration of cholesterol within small-sized and medium-sized LDL subfractions was reduced post-treatment $(P<0.05)$. LDL particle size was associated with reduced body weight $(r-0.32, P=0.04)$ and smaller waist circumference post-treatment $(r-0.36, P=0.03)$. No relationship between post-treatment LDL particle size and TAG $(r-0 \cdot 28$, $P=0 \cdot 11)$ or HDL-cholesterol concentrations ( $r \quad 0 \cdot 18$, $P=0.65$ ) was observed.

\section{Discussion}

ER-induced weight loss is an effective means of increasing LDL particle size in obese adults. Short-term studies in this area (12-24 weeks in duration) generally report increases in peak LDL particle size $(0 \cdot 5-3 \AA$ increase from baseline) with 5-9 kg of weight loss in obese men and women $^{(4-6,13)}$, with some exceptions ${ }^{(14,15)}$. The ability of fasting regimens, such as ADMF, to exert similar beneficial effects as ER, had never been tested. We show here, for the first time, that ADMF is able to increase peak LDL particle size ( $2 \AA$ increase from baseline) to a similar extent as ER. Whether one of these dietary restriction regimens is more effective in increasing LDL particle size is not clear at present, due to the limited amount of ADMF data presently available. Future studies are needed to clarify which dietary restriction regimen is superior with regard to increasing LDL particle size. In addition to these changes in peak particle size, we also observed a distinct decrease in the proportion of small particles (13-9\%) and a distinct increase in the proportion of large LDL particles (68-76\%) after 8 weeks of treatment. Reductions in plasma LDLcholesterol and TAG concentrations were also observed.

Table 1. LDL particle size and distribution over the course of the trial

(Mean values with their standard errors, $n 16)^{*}$

\begin{tabular}{|c|c|c|c|c|c|c|c|c|}
\hline & \multicolumn{4}{|c|}{ Pre-loss control phase } & \multirow{2}{*}{\multicolumn{2}{|c|}{$\begin{array}{c}\text { ADMF controlled } \\
\text { feeding phase }\end{array}$}} & \multirow{2}{*}{\multicolumn{2}{|c|}{$\begin{array}{c}\begin{array}{c}\text { ADMF self-selected } \\
\text { feeding phase }\end{array} \\
\text { Days } 69,70\end{array}$}} \\
\hline & \multicolumn{2}{|c|}{ Day 1} & \multicolumn{2}{|c|}{ Day 14} & & & & \\
\hline & Mean & SEM & Mean & SEM & Mean & SEM & Mean & SEM \\
\hline LDL peak particle size $(\AA)$ & $266^{a}$ & 1 & $266^{a, b}$ & 1 & $267^{a, b}$ & 1 & $268^{\mathrm{b}}$ & 1 \\
\hline LDL integrated particle size $(\AA)$ & $267^{\mathrm{a}}$ & 2 & $268^{a, b}$ & 2 & $267^{a, b}$ & 1 & $269^{b}$ & 2 \\
\hline $\mathrm{LDL}_{>260 \AA}(\%)$ & $68^{a}$ & 4 & $71^{a, b}$ & 5 & $73^{a, b}$ & 4 & $76^{\mathrm{b}}$ & 4 \\
\hline $\mathrm{LDL}_{260-255 \AA}(\%)$ & $19^{a}$ & 2 & $17^{\mathrm{a}, \mathrm{b}}$ & 2 & $16^{a, b}$ & 2 & $15^{\mathrm{b}}$ & 2 \\
\hline $\mathrm{LDL}_{<255 \AA}(\%)$ & $13^{a}$ & 2 & $12^{\mathrm{a}, \mathrm{b}}$ & 3 & $11^{\mathrm{a}, \mathrm{b}}$ & 2 & $9^{b}$ & 3 \\
\hline LDL-C $>260 \AA(\mathrm{mg} / \mathrm{l})$ & 700 & 90 & 780 & 10 & 520 & 70 & 490 & 70 \\
\hline LDL- $C_{260-255 \AA}(\mathrm{mg} / \mathrm{l})$ & $190^{\mathrm{a}}$ & 30 & $170^{\mathrm{a}}$ & 20 & $120^{a, b}$ & 20 & $100^{\mathrm{b}}$ & 10 \\
\hline $\mathrm{LDL}_{-\mathrm{C}}<255 \AA$ ( $\left.\mathrm{mg} / \mathrm{l}\right)$ & $130^{\mathrm{a}}$ & 20 & $110^{a, b}$ & 30 & $80^{a, b}$ & 20 & $50^{\mathrm{b}}$ & 20 \\
\hline
\end{tabular}

ADMF, alternate day modified fasting; LDL-C, LDL-cholesterol.

${ }_{a, b}$ Mean values within a row (for each LDL particle size parameter) with unlike superscript letters were significantly different (one-factor ANOVA with Bonferroni analysis, $P<0.05)$.

*Days 41 and 42 values combined; days 69 and 70 values combined. 
However, the present study is limited in that the effect of weight loss on LDL particle size was not evaluated separately from the effect of fasting. Therefore, it is not clear whether these cardioprotective effects were mediated by reduced body weight or by the prolonged fasting period on the fast day. Future studies in this area, which administer the ADMF regimen with and without a prolonged fasting period, are necessary to tease apart the effects of these variables on changes in lipoprotein subfractions.

A secondary aim of the present study was to evaluate how modulations in LDL diameter by ADMF are related to changes in abdominal fat mass and plasma lipid concentrations. Increased waist circumference is associated with augmented plasma TAG, which in turn is associated with decreases in LDL particle size ${ }^{(16)}$. We show here that 8 weeks of ADMF is able to decrease the waist circumference by $4.0(\operatorname{sem} 0.9) \mathrm{cm}$, and that this smaller waist circumference is associated with increased LDL particle size. Similar relationships have also been reported in short-term ER trials $^{(3,17)}$. However, no relationship was noted between post-treatment TAG concentrations and waist circumference. Moreover, no association was observed between post-treatment TAG concentrations and LDL particle size. A possible reason for this lack of relationship may be the small sample size used here. If a larger number of subjects had been recruited, it is probable that these relationships might have been statistically significant.

In summary, these findings suggest that short-term ADMF may be an effective strategy for increasing LDL particle size and decreasing the proportion of small, dense LDL. These data support the growing body of evidence advocating $\mathrm{ADMF}$ as a suitable alternative to traditional ER for lowering heart disease risk in obese adults.

\section{Acknowledgements}

The authors' responsibilities were as follows: K. A. V. designed the study, oversaw the data collection and wrote the manuscript; S. B. conducted the clinical trial; M. C. K. performed the laboratory analyses; B. L. assisted with the interpretation of the data and in the writing of the manuscript. The authors have no conflicts of interest to report. The present study was funded by the Department of Kinesiology and Nutrition at the University of Illinois, Chicago.

\section{References}

1. Griffin BA, Freeman DJ, Tait GW, et al. (1994) Role of plasma triglyceride in the regulation of plasma low density lipoprotein (LDL) subfractions: relative contribution of small, dense
LDL to coronary heart disease risk. Atherosclerosis 106 241-253.

2. Superko HR \& Gadesam RR (2008) Is it LDL particle size or number that correlates with risk for cardiovascular disease? Curr Atheroscler Rep 10, 377-385.

3. Despres JP (2007) Cardiovascular disease under the influence of excess visceral fat. Crit Pathw Cardiol 6, 51-59.

4. Morgan LM, Griffin BA, Millward DJ, et al. (2009) Comparison of the effects of four commercially available weight-loss programmes on lipid-based cardiovascular risk factors. Public Health Nutr 12, 799-807.

5. Krauss RM, Blanche PJ, Rawlings RS, et al. (2006) Separate effects of reduced carbohydrate intake and weight loss on atherogenic dyslipidemia. Am J Clin Nutr 83, 1025-1031.

6. Westman EC, Yancy WS Jr, Olsen MK, et al. (2006) Effect of a low-carbohydrate, ketogenic diet program compared to a low-fat diet on fasting lipoprotein subclasses. Int J Cardiol 110, 212-216.

7. Varady KA, Bhutani S, Church EC, et al. (2009) Short-term modified alternate-day fasting: a novel dietary strategy for weight loss and cardioprotection in obese adults. Am J Clin Nutr 90, 1138-1143.

8. Johnson JB, Summer W, Cutler RG, et al. (2007) Alternate day calorie restriction improves clinical findings and reduces markers of oxidative stress and inflammation in overweight adults with moderate asthma. Free Radic Biol Med $\mathbf{4 2}$ 665-674.

9. Mifflin MD, St Jeor ST, Hill LA, et al. (1990) A new predictive equation for resting energy expenditure in healthy individuals. Am J Clin Nutr 51, 241-247.

10. Friedewald WT, Levy RI \& Fredrickson DS (1972) Estimation of the concentration of low-density lipoprotein cholesterol in plasma, without use of the preparative ultracentrifuge. Clin Chem 18, 499-502.

11. St-Pierre AC, Ruel IL, Cantin B, et al. (2001) Comparison of various electrophoretic characteristics of LDL particles and their relationship to the risk of ischemic heart disease. Circulation 104, 2295-2299.

12. Tchernof A, Lamarche B, Prud'Homme D, et al. (1996) The dense LDL phenotype. Association with plasma lipoprotein levels, visceral obesity, and hyperinsulinemia in men. Diabetes Care 19, 629-637.

13. Wood RJ, Volek JS, Liu Y, et al. (2006) Carbohydrate restriction alters lipoprotein metabolism by modifying VLDL, LDL, and HDL subfraction distribution and size in overweight men. J Nutr 136, 384-389.

14. Harman NL, Leeds AR \& Griffin BA (2008) Increased dietary cholesterol does not increase plasma low density lipoprotein when accompanied by an energy-restricted diet and weight loss. Eur J Nutr 47, 287-293.

15. Tzotzas T, Filippatos TD, Triantos A, et al. (2008) Effects of a low-calorie diet associated with weight loss on lipoproteinassociated phospholipase $\mathrm{A}_{2}$ (Lp-PLA $)$ activity in healthy obese women. Nutr Metab Cardiovasc Dis 18, 477-482.

16. Rainwater DL, Mitchell BD, Comuzzie AG, et al. (1999) Relationship of low-density lipoprotein particle size and measures of adiposity. Int J Obes Relat Metab Disord 23, 180-189.

17. Clifton PM, Noakes M \& Nestel PJ (1998) LDL particle size and LDL and HDL cholesterol changes with dietary fat and cholesterol in healthy subjects. J Lipid Res 39, 1799-1804. 